\title{
Muscarinic M2 Receptors on Peripheral Nerve Endings: A Molecular Target of Antinociception
}

\author{
Nadia Bernardini, ${ }^{1}$ Carolina Roza, ${ }^{1}$ Susanne K. Sauer, ${ }^{1}$ Jesus Gomeza, ${ }^{2}$ Jürgen Wess, ${ }^{2}$ and Peter W. Reeh ${ }^{1}$ \\ ${ }^{1}$ Department of Physiology and Experimental Pathophysiology, University of Erlangen, D-91054 Erlangen, Germany, and \\ 2Laboratory of Bioorganic Chemistry, National Institute of Diabetes and Digestive and Kidney Diseases, Bethesda, \\ Maryland 20892
}

\begin{abstract}
We recently described a novel endogenous mechanism of peripheral antinociception, possibly involving activation of muscarinic M2 acetylcholine receptors that are expressed on nociceptive nerve endings and decrease their sensitivity. In the present study, this mechanism was scrutinized in skin taken from mice with targeted deletions of the muscarinic M2 receptor gene and, for control purposes, of the M4 receptor gene. Two different approaches were taken. Electrophysiologically the effects of muscarine on nociceptive afferents were investigated using the mouse skin-saphenous nerve preparation, in vitro. Muscarine did not excite nociceptors in the wild-type littermates (WT) and M4 knock-out (M4 KO) mice, but almost all fibers exhibited marked desensitization to mechanical and heat stimuli. Surprisingly, in the M2 KO mice, muscarine was able to excite C-nociceptors and to induce a mild sensitization to heat but caused no alteration in mechanical responsiveness tested
\end{abstract}

Muscarinic acetylcholine receptors are known to be involved in the control of many peripheral as well as central cholinergic responses (Caulfield, 1993). Five different muscarinic receptor subtypes (M1-M5) have been identified and characterized (Bonner et al., 1988, 1989), all of which are members of the G-proteincoupled receptor superfamily. The M1, M3, and M5 receptors act preferentially through stimulation of the phosphoinositol cascade (via $\mathrm{G}_{\mathrm{q}} / \mathrm{G}_{11}$ ), whereas the $\mathrm{M} 2$ and $\mathrm{M} 4$ receptors mainly mediate the inhibition of adenylyl cyclase (via $\mathrm{G}_{\mathrm{i}} / \mathrm{G}_{0}$ ) (Caulfield and Birdsall, 1998). Many studies reported the presence of muscarinic receptors on peripheral nociceptors (Steen and Reeh, 1993; Wanke et al., 1994; Bernardini et al., 1999, 2001a,b; Haberberger et al., 1999; Tata et al., 2000), although their involvement in pain control mechanisms was not proven until recently. In an electrophysiological study, we demonstrated previously that muscarine treatment of polymodal nociceptors left almost all fibers with a marked and sustained desensitization to mechanical and heat stimulation (Bernardini et al. 2001b). This effect could be prevented by the non-subtype-selective muscarinic antagonist scopolamine and by the M2-preferring antagonist gallamine; moreover, the M2-preferring agonist arecaidine mimicked the

\footnotetext{
Received Nov. 27, 2001; revised March 18, 2002; accepted March 25, 2002.

This work was supported by Deutsche Forschungsgemeinschaft, SFB 353-B12. C.R. held a postdoctoral fellowship from the Ministry of Education and Culture of Spain. We thank I. Izydorczyk and A. Kuhn for technical assistance.

Correspondence should be addressed to Dr. P. W. Reeh, Department of Physiology and Experimental Pathophysiology, University of Erlangen, Universitätstrasse 17, D-91054 Erlangen, Germany. E-mail: reeh@physiologie1.unierlangen.de.

Copyright (C) 2002 Society for Neuroscience $\quad 0270-6474 / 02 / 220001-\bullet \$ 15.00 / 0$
}

with von Frey hairs. In the second, neurochemical approach, the heat-induced cutaneous release of calcitonin gene-related peptide (CGRP) was investigated to gain comparative data on the neurosecretory (vasodilatory) functions of the primary afferent neurons. The substantial increase of CGRP release evoked by noxious heat $\left(47^{\circ} \mathrm{C}\right)$ was diminished under muscarine by $>50 \%$ in the WT and M4 KO animals but remained unaltered in the M2 KO mice. Together, these data provide direct evidence that $\mathrm{M} 2$ receptors on cutaneous nerve endings mediate effective depression of nociceptive responsiveness. This observation should be of interest for the development of novel classes of analgesic agents.

Key words: primary afferents; cholinergic; desensitization; noxious heat; mechanosensitivity; M2 knock-out; M4 knockout; pain; analgesia

desensitizing effect of muscarine. In a further study, we demonstrated that muscarine and arecaidine dose-dependently decreased the basal and heat-induced CGRP release from isolated rat skin (Bernardini et al., 2001a). Together, these data suggest an involvement of the M2 receptor in muscarinic nociceptor desensitization and in control of "neurogenic inflammation." However, the lack of muscarinic ligands with a high degree of receptor subtype selectivity and the fact that primary sensory neurons co-express more than one muscarinic receptor subtype (Bernardini et al., 1999; Tata et al., 2000) make it difficult to conclusively assign specific functional responses to individual receptor subtypes.

Thus, the availability of mutant mice that lack either M2 or M4 receptors (Gomeza et al., 1999a,b) has provided the opportunity to scrutinize the physiological role of muscarinic receptors in peripheral nociception in a more direct manner. Recent studies with M2 and M4 receptor knock-out (KO) mice had already

This article is published in The Journal of Neuroscience, Rapid Communications Section, which publishes brief, peerreviewed papers online, not in print. Rapid Communications are posted online approximately one month earlier than they would appear if printed. They are listed in the Table of Contents of the next open issue of JNeurosci. Cite this article as: JNeurosci, 2002, 22:RC229 (1-5). The publication date is the date of posting online at www.jneurosci.org.

http://www.jneurosci.org/cgi/content/full/6476 
revealed a crucial role of the muscarinic M2 receptor subtype in mediating the central analgesic effects of oxotremorine, as assessed in the hot plate and the tail-flick tests (Gomeza et al., 1999a). The present electrophysiological and neurochemical studies focused on comparing the effects of muscarine on peripheral nociceptors in skin prepared from M2 and M4 receptor $\mathrm{KO}$ mice and their WT littermates.

\section{MATERIALS AND METHODS}

Animals. The generation of M2 and M4 muscarinic receptor knock-out mice has been described previously (Gomeza et al. 1999a,b). The M2 KO and the corresponding WT mice are 129"J1"/CF-1 hybrids, whereas the M4 KO and the corresponding WT mice are 129SvEvTac/CF-1 hybrids. For this study we used 4- to 8-week-old male mice from the F3 generation.

Electrophysiology. This study was performed using the in vitro skinsaphenous nerve preparation that has been described previously in detail (Reeh, 1986, 1988).

The preparations were obtained from M2 KO and WT (three each) and M4 KO and WT mice (four each), killed in a pure $\mathrm{CO}_{2}$ atmosphere. The saphenous nerve in continuity with the dorsal hindpaw skin was subcutaneously dissected and excised. The skin was pinned out, corium side up, in a Perspex chamber and kept under laminar superfusion (16 $\mathrm{ml} / \mathrm{min}$ ). The saphenous nerve was pulled through a hole into a second chamber where the aqueous solution was overlaid with paraffin oil; here, the nerve was subdivided into smaller and smaller filaments until singlefiber unitary activity could be recorded via gold wire electrodes.

The skin was superfused with "synthetic interstitial fluid" (SIF) containing (in mM): $108 \mathrm{NaCl}, 3.48 \mathrm{KCl}, 3.5 \mathrm{MgSO}_{4}, 26 \mathrm{NaHCO}_{3}, 1.7$ $\mathrm{NaH}_{2} \mathrm{PO}_{4}, 1.5 \mathrm{CaCl}_{2}, 9.6$ sodium gluconate, 5.55 glucose, 7.6 sucrose (Bretag, 1969) at $32^{\circ} \mathrm{C}$.

Receptive fields of $\mathrm{C}$-fibers were searched for by probing the corium side of the skin with a blunt glass rod. The nerve endings were electrically stimulated in their receptive fields via Teflon-insulated steel microelectrodes (impedance 6-10 $\mathrm{M} \Omega$ ) to measure conduction velocity and establish the identity of mechanically and electrically evoked impulses using the "marking phenomenon" (Weidner et al., 1999). The thresholds to mechanical stimulation were tested with a set of 17 von Frey hairs calibrated from 1 to $256 \mathrm{mN}$ in a geometric series $\left(x_{\mathrm{i}}=x_{\mathrm{i}-1} * \sqrt{ } 2\right)$. Heat responsiveness was examined by focusing a halogen lamp through the translucent bottom of the skin chamber onto the epidermal side of the isolated receptive field. At the opposite corium side, the linearly increasing temperature (from 32 to $46^{\circ} \mathrm{C}$ in $20 \mathrm{sec}$, which corresponds to a peak temperature of $52^{\circ} \mathrm{C}$ at the epidermal surface) (Reeh, 1986) was feedback controlled by a thermocouple. To isolate the receptive field, a metal ring was placed over the respective corium area, and the SIF content was evacuated. The temperature corresponding to the second spike of the heat response of a fiber was considered as heat threshold. Heat and mechanical testing were always performed before and after drug administration (i.e., at a $6 \mathrm{~min}$ interval).

The metal rings to isolate receptive fields were also used for chemical stimulations. Muscarine $10^{-4} \mathrm{M}$ in SIF was applied to the receptive fields for $5 \mathrm{~min}$, and then the mechanical and heat thresholds were redetermined.

The single nerve fiber activity was recorded with a low-noise ACcoupled amplifier and monitored on a loudspeaker and an oscilloscope. The recordings were digitized and processed in an AT-type computer using a DAP 1200 interface card (Microstar, Redmond, WA). The data were analyzed off-line using the Spike/Spidi software package that provides a template-matching procedure for automatic spike discrimination (Forster and Handwerker, 1990).

The magnitude of a chemical or heat response was assessed as the total number of spikes counted during the $5 \mathrm{~min}$ or $20 \mathrm{sec}$ of stimulation, respectively.

CGRP release. We used $\mathrm{M} 2 \mathrm{KO}$ and $\mathrm{WT}$ (nine each) and $\mathrm{M} 4 \mathrm{KO}$ and WT mice (eight each). Animals were euthanized by exposure to a pure $\mathrm{CO}_{2}$ atmosphere, and the hairy skin from both hindpaws was subcutaneously dissected from below the knee. The skin flaps obtained $(n=68)$ had an average weight of $\sim 100 \mathrm{mg}$ (range $80-120 \mathrm{mg}$ ); they were wrapped around acrylic glass rods $(\varnothing=6 \mathrm{~mm})$ with the corium side exposed and fixed with surgical silk. The preparations were washed for 30 min in SIF. A series of four or five consecutive glass tubes were filled with $1 \mathrm{ml} \mathrm{SIF}$, which had been previously bubbled with carbogen $(95 \%$
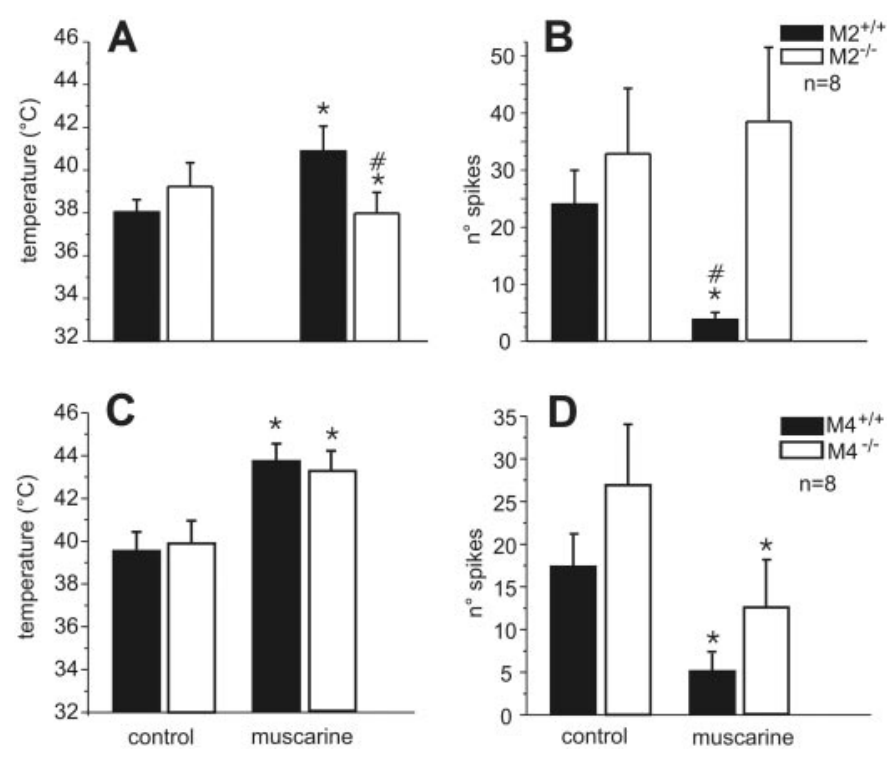

Figure 1. Heat responsiveness. Mean heat-induced discharge $(B, D)$ and heat threshold $(A, C)$ of primary afferent $\mathrm{C}$-MH fibers before (control) and after administration of muscarine $10^{-4} \mathrm{M}$ for $5 \mathrm{~min}$ to the receptive fields in the isolated skin-nerve preparation. $n$ refers to the number of units tested. Asterisks indicate the intragroup significance (*Wilcoxon matched pairs test comparing to the control heat response), and the \# symbol indicates significance between $\mathrm{KO}(-/-)$ mice and the corresponding WT $(+/+)$ littermates (\# Mann-Whitney $U$ test).

$\left.\mathrm{O}_{2}, 5 \% \mathrm{CO}_{2}\right)$, and positioned in a shaking bath $\left(32^{\circ} \mathrm{C}\right)$. The skin flaps were incubated for $5 \mathrm{~min}$ in the first test tube and then forwarded to the next tube for $5 \mathrm{~min}$ and so forth; the third tube always contained the stimulating solution on the basis of SIF at $47^{\circ} \mathrm{C}$. Muscarine chloride $10^{-4} \mathrm{M}$ (Sigma) was added to the heated solution. Both skin flaps of one animal were always examined in parallel; one side was control, and the other was treated with muscarine.

The CGRP content of the incubation fluid was measured using commercial enzyme immunoassays (EIAs) (Cayman Chemical, Ann Arbor, MI; distributed through SPIbi, Massy, France) immediately after the experiment as described in detail (Averbeck and Reeh, 2001). All EIA plates were determined photometrically using a microplate reader (Dynatech Alexandria, VA).

The values of CGRP were calculated referring to $1 \mathrm{gm}$ fresh weight of skin. Average results are given as mean \pm SEM. In the figures, $n$ refers to the number of different animals used. For better comparison of effect magnitudes, we used normalized values in the figures. For this, the actual value was divided by the value of the second baseline sample, which was the last one collected before stimulation. Nonparametric statistical comparisons within groups of identical experiments were made using the Wilcoxon matched pairs test, and the Mann-Whitney $U$ test was used to compare among different groups. Differences were considered statistically significant at $p<0.05$.

\section{RESULTS}

\section{Single fiber recordings}

Altogether, we examined 32 mechano-heat-sensitive C-fibers (C$\mathrm{MH}$ ), eight units from each of the four groups of experimental animals. Conduction velocities ranged from $0.3 \mathrm{~m} / \mathrm{sec}$ to 0.5 $\mathrm{m} / \mathrm{sec}$; no fiber showed spontaneous activity. All receptive fields were tested with muscarine at $10^{-4} \mathrm{M}$ concentration, which is a supramaximal concentration with respect to nociceptor desensitization (Bernardini et al., 2001b).

\section{$M 2 W T$}

All units revealed a marked and significant desensitization against heat stimulation after muscarine administration (Fig. $1 A, B)$. The vast majority of the $\mathrm{C}-\mathrm{MH}$ fibers showed a gradual 

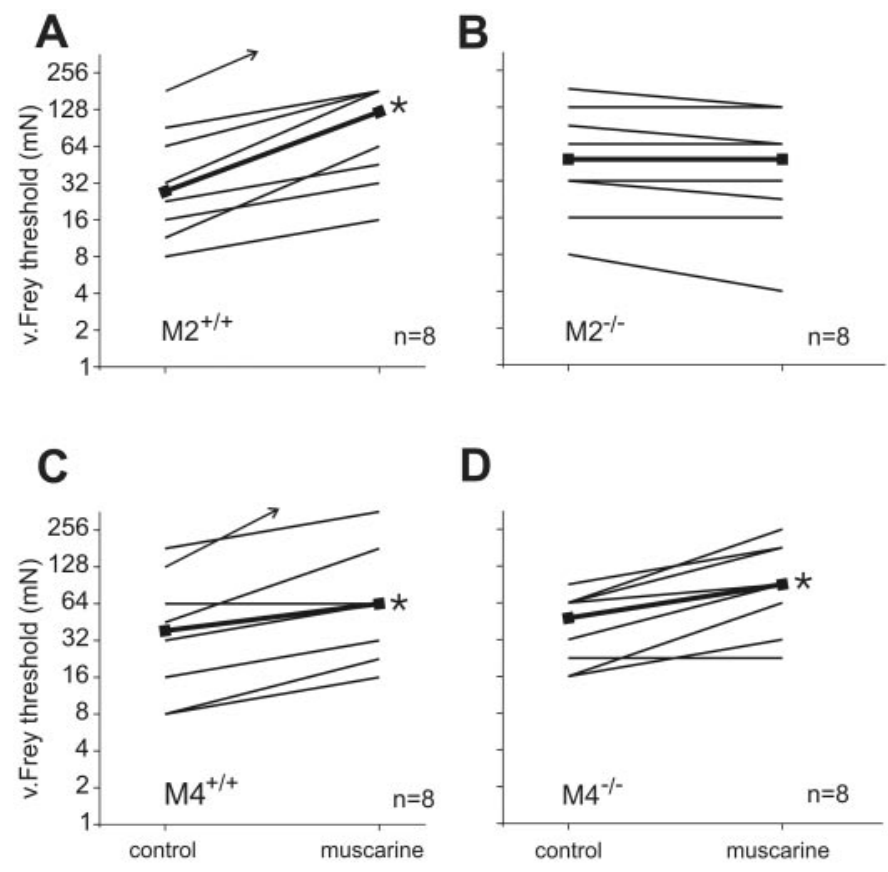

Figure 2. Von Frey thresholds. Mechanical thresholds tested with von Frey hairs before (control) and after administration of muscarine $\left(10^{-4}\right.$ M) to the receptive fields of the C-MH fibers. Arrowheads in $A$ and $C$ indicate nociceptive nerve fibers responding only to glass rod pressure $(\sim 1000 \mathrm{mN})$. The bold lines indicate the median von Frey thresholds. Asterisks indicate the intragroup significance (*Wilcoxon matched pairs test).

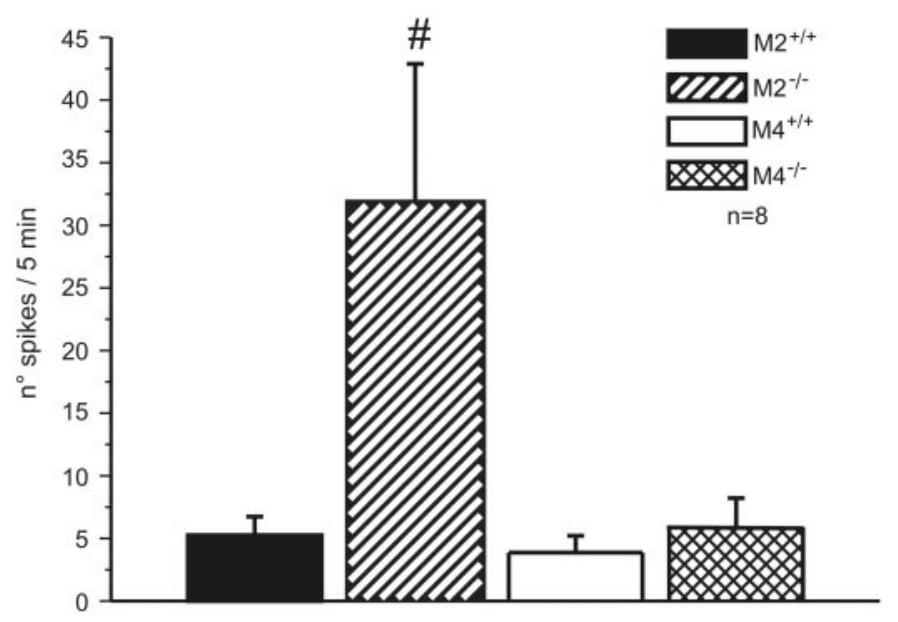

Figure 3. Muscarine-induced excitation. Cutaneous C-MH fiber discharge during $5 \mathrm{~min}$ of muscarine $\left(10^{-4} \mathrm{M}\right)$ treatment. $n$ refers to the number of fibers examined. The \# symbol indicates a significant difference between the M2 KO mice $(-/-)$ and the other groups (\# Mann-Whitney $U$ test).

increase of the heat threshold; one was no longer excited by heat stimulation up to $46^{\circ} \mathrm{C}$, but its heat responsiveness recovered after a 20 min washout period (data not shown). The increase of the heat threshold was always accompanied by a decrease in the total number of spikes during heat stimulation. The fibers tested were also significantly desensitized by muscarine to mechanical stimulation (Fig. 2A). One of the eight fibers tested responded only to probing of the receptive field with a blunt glass rod applying $\sim 1000 \mathrm{mN}$ of force (Fig. 2A, arrow).
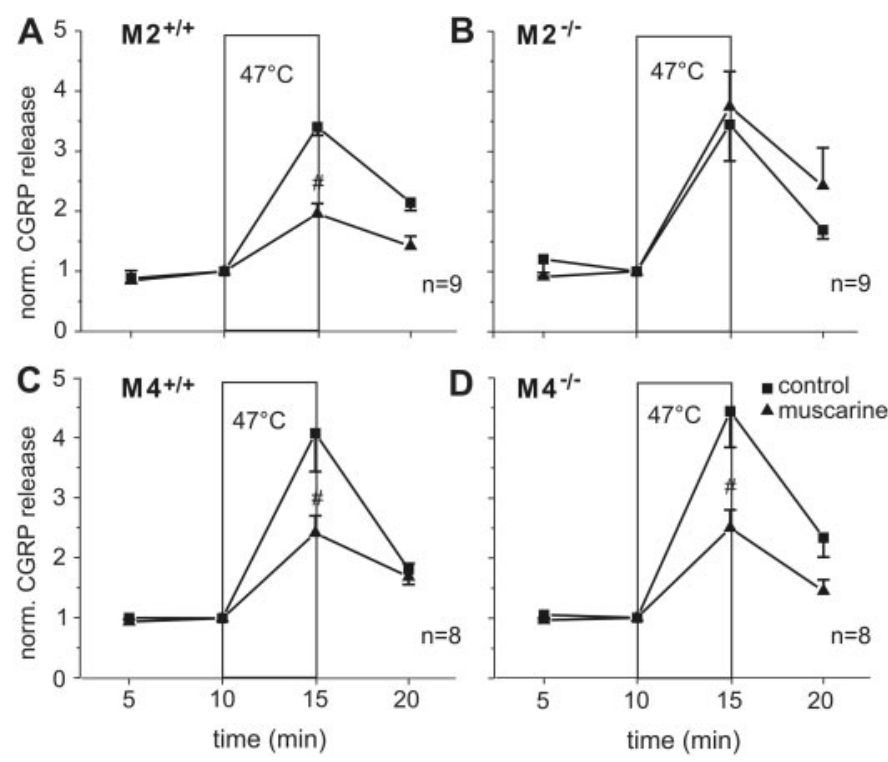

Figure 4. Muscarine effects on heat-induced CGRP release from isolated skin. Muscarine $10^{-4} \mathrm{M}$ had an inhibitory effect on CGRP release in the $\mathrm{M} 2^{+/+}(A), \mathrm{M} 4^{+/+}(C)$, and $\mathrm{M} 4^{-1-}$ mice $(D)$ but not in the $\mathrm{M} 2^{-1-}$ mice $(B)$. The open columns indicate the period of stimulation with heated SIF and coapplication of muscarine; $n$ refers to the number of preparations from different animals. All increases over baseline were significant (Wilcoxon matched pairs test), and the \# symbols indicate significant differences between groups (\#Mann-Whitney $U$ test).

\section{$\mathrm{M} 2 \mathrm{KO}$}

Interestingly, many of the $\mathrm{C}$-MH fibers were weakly and transiently excited during muscarine administration, showing an enhanced mean discharge rate that was significantly higher than in the other experimental groups (Fig. 3). Moreover, five of the eight $\mathrm{C}-\mathrm{MH}$ fibers tested were actually sensitized against heat stimulation; the whole group showed a significant decrease of the mean heat threshold and an increase of the heat-induced discharge that was not significant (Fig. 1 $A, B$ ). On the contrary, there was no alteration of the median von Frey threshold (Fig. 2B).

\section{M4 WT}

All tested fibers showed a clear and significant desensitization to heat stimulation after muscarine administration (Fig. $1 C, D$ ), and two units no longer responded to heat stimulation up to $46^{\circ} \mathrm{C}$, but they were able to recover their heat responsiveness after a 10-20 min washout period (data not shown). In all but one of the units we also found a marked desensitization to mechanical stimulation (Fig. $2 C$ ), and one fiber responded only to probing the receptive field with a glass rod $(\sim 1000 \mathrm{mN})$; the same unit no longer responded to heat stimulation but was still electrically excitable (Fig. 2C, arrow).

\section{$\mathrm{M} 4 \mathrm{KO}$}

Seven of eight units examined were desensitized against heat stimulation after muscarine administration, showing a significant increase in the mean heat threshold and a parallel decrease in the number of spikes (Fig. 1C,D). One fiber did not respond to heat stimulation up to $46^{\circ} \mathrm{C}$. The median von Frey threshold was significantly increased (Fig. 2D).

\section{Muscarinic effects on heat-induced CGRP release}

The basal CGRP release from all experiments amounted to $93 \pm$ $4 \mathrm{pg} / \mathrm{gm}$ fresh weight of skin; the means of the four experimental groups did not differ significantly. 
Muscarine $10^{-4} \mathrm{M}$ significantly reduced the mean heat-induced CGRP release by $57 \%$ in the M2 WT mice but was totally ineffective in decreasing neuropeptide release in the $\mathrm{M} 2 \mathrm{KO}$ mice (Fig. $4 A, B$ ). In preparations from M4 KO and M4 WT mice, muscarine suppressed heat-induced CGRP release by 56 and $59 \%$, respectively, similar to the results obtained with M2 WT mice (Fig. $4 C, D$ ).

\section{DISCUSSION}

In the present study we have analyzed the effects of muscarine on peripheral nociceptors of mice lacking M2 or M4 receptors and of their WT littermates (Gomeza et al., 1999a,b). Muscarine treatment induced major desensitization to mechanical and noxious heat stimuli of cutaneous C-units in the WT mice, in agreement with previous data gained from the same isolated skin preparation in the rat (Steen and Reeh, 1993; Bernardini et al., 2001b). On the basis of studies with muscarinic agonists and antagonists, we recently proposed that these effects might involve activation of M2 receptors (Bernardini et al., 2001b). However, the lack of muscarinic ligands endowed with a high degree of receptor subtype selectivity left this proposal somewhat uncertain, in particular with respect to the differentiation between M2 and M4 receptors, which both couple to G-proteins of the $G_{i}$ family (Wess, 1996; Caulfield and Birdsall, 1998).

In the present work we showed that the desensitizing effects of muscarine were unaltered in mice lacking the M4 receptor, excluding this muscarinic receptor subtype as a mediator of peripheral antinociception. However, in the M2 KO mice, muscarine was no longer able to cause reductions in nociceptor sensitivity. On the contrary, in these animals, muscarine induced a lowtransient discharge in the $\mathrm{C}$-fibers and a mild sensitization of the heat responsiveness. This effect is unlikely to be due to the repeated application of moderate heat stimuli (Reeh, 1988; Steen and Reeh, 1993; Guenther et al., 1999; Bernardini et al., 2001b) but may be caused by the presence of other muscarinic receptor subtypes, such as M1 and M3, on sensory neurons (Bernardini et al., 1999; Tata et al., 2000). Although M3 receptors were not found on rat cutaneous nerve terminals via immunocytochemistry (Bernardini et al., 2001b), such receptors are expressed in cultured rat dorsal root ganglion neurons and induce a substantial $\mathrm{Ca}^{2+}$ influx during activation by muscarine (Haberberger et al., 2000). An increase in intracellular $\mathrm{Ca}^{2+}$ can induce nociceptor sensitization (Guenther et al., 1999), and this pathway may be particularly effective in the absence of the desensitizing M2 receptors. The electrophysiological results are well supported by the neurochemical findings showing that muscarine depressed stimulated CGRP release in WT and M4 KO mice but not in M2 $\mathrm{KO}$ mice.

These results clearly indicate that M2 receptors are responsible for cholinergic nociceptor desensitization. There might be several possible sources of peripheral, endogenous acetylcholine. In fact, it has been demonstrated that sensory neurons themselves express choline acetyltranferase and acetylcholinesterase and are able to synthesize acetylcholine (ACh) (Tata et al., 1994). Moreover, an increasing number of reports indicates a rather widespread expression of cholinergic markers in non-neuronal cells. In particular, human keratinocytes as well as fibroblasts and glial cells synthesize and release ACh (Grando et al., 1993; Wessler et al., 1997; Buchli et al., 1999). Keratinocytes are a source of continuous ACh release in the skin (Grando et al., 1993; Nguyen et al., 2001), in the closest possible vicinity to epidermal nerve endings equipped with M2 receptors (Bernardini et al., 2001b). However, local tissue concentrations of $\mathrm{ACh}$ in the skin in health and disease are unknown. Nevertheless, one might speculate that nociceptor sensitivity is normally under permanent inhibitory control through tonic activation of $\mathrm{M} 2$ receptors, a concept that may have important pathophysiological implications.

Cholinergic desensitization of nociceptors has previously been found to be sustained and long lasting (e.g., for at least $40 \mathrm{~min}$ after 5 min exposure to agonists) (Steen and Reeh, 1993; Bernardini et al. 2001b). Moreover, it has been reported recently that cholinesterase blockers can prevent post-arthroscopy pain when instillated in the knee joint to increase ACh levels in the synovial fluid (Buerkle et al., 1998; Yang et al., 1998). Our present data suggest that this analgesic activity mostly likely involves AChmediated activation of peripheral M2 receptors. Because stimulation of M2 receptors results in reduced pain sensitivity through both peripheral (this study) and central mechanisms (Gomeza et al. 1999a), agents that can selectively activate this receptor subtype should have considerable therapeutic potential.

\section{REFERENCES}

Averbeck B, Reeh PW (2001) Interactions of inflammatory mediators stimulating release of calcitonin gene-related peptide, substance $\mathrm{P}$, prostaglandin $\mathrm{E}(2)$ from isolated rat skin. Neuropharmacology 40:416-423.

Bernardini N, Levey AI, Augusti-Tocco G (1999) Rat dorsal root ganglia express $\mathrm{m} 1-\mathrm{m} 4$ muscarinic receptor proteins. J Peripher Nerv Syst 4:222-232.

Bernardini N, Reeh PW, Sauer SK (2001a) M2 receptors inhibit heatinduced CGRP release from isolated rat skin, in vitro. NeuroReport 12:2457-2460.

Bernardini N, Sauer SK, Haberberger R, Fischer MJM, Reeh PW (2001b) Excitatory nicotinic and desensitizing muscarinic (M2) effects on C-nociceptors in isolated rat skin. J Neurosci 21:3295-3302.

Bonner T (1989) The molecular basis of muscarinic acetylcholine receptor diversity. Trends Neurosci 12:148-151.

Bonner T, Young A, Brann M (1988) Cloning and expression of the human and rat $\mathrm{m} 5$ muscarinic acetylcholine receptor genes. Neuron 1:403-410.

Bretag A (1969) Synthetic interstitial fluid for isolated mammalian tissue. Life Sci 8:319-329.

Buchli R, Ndoye A, Rodriguez JG, Zia S, Webber RJ, Grando SA (1999) Human skin fibroblasts express $\mathrm{m} 2, \mathrm{~m} 4$, and $\mathrm{m} 5$ subtypes of muscarinic acetylcholine receptors. J Cell Biochem 74:264-277.

Buerkle H, Boschin M, Marcus MA, Brodner G, Wusten R, Van Aken H (1998) Central and peripheral analgesia mediated by the acetylcholinesterase-inhibitor neostigmine in rat inflamed knee joint model. Anesth Analg 86:1027-1032.

Caulfield MP (1993) Muscarinic receptors: characterization, coupling and function. Pharmacol Ther 58:319-379.

Caulfield MP, Birdsall NGM (1998) International union pharmacology. XVII. Classification of muscarinic acetylcholine receptors. Pharmacol Rev 50:279-290.

Forster C, Handwerker HO (1990) Automatic classification and analysis of microneurographic spike data using a PC/AT. J Neurosci Methods 31:109-118.

Gomeza J, Shannon H, Kostenis E, Felder C, Zhang L, Brodkin J, Grinberg A, Sheng H, Wess J (1999a) Pronounced pharmacological deficits in M2 muscarinic acetylcholine receptor knockout mice. Proc Natl Acad Sci USA 96:1692-1697.

Gomeza J, Zhang L, Kostenis E, Felder C, Bymaster F, Brodkin J, Shannon H, Xia B, Deng C, Wess J (1999b) Enhancement of D1 dopamine receptor-mediated locomotor stimulation in $\mathrm{M}_{4}$ muscarinic acetylcholine receptor knockout mice. Proc Natl Acad Sci USA 96:12222-12223.

Grando SA, Kist DA QiM, Dahl MV (1993) Human keratinocytes synthesize, secrete and degrade acetylcholine. J Invest Dermatol 101:32-36.

Guenther S, Reeh PW, Kress M (1999) Rises in $\left[\mathrm{Ca}^{2+}\right]_{i}$ mediate capsaicin- and proton-induced heat sensitization of rat primary nociceptive neurons. Eur J Neurosci 11:3143-3150.

Haberberger R, Henrich M, Couraud JY, Kummer W (1999) Muscarinic M2-receptors in rat thoracic dorsal root ganglia. Neurosci Lett 266:177-180.

Haberberger R, Scholz R, Kummer W, Kress M (2000) M2-receptor subtype does not mediate muscarine-induced increases in $\left[\mathrm{Ca}^{++}\right]_{\mathrm{i}}$ in nociceptive neurons of rat dorsal root ganglia. J Neurophysiol 84:1934-1941. 
Nguyen VT, Ndnoye A, Hall LL, Zia S, Arredondo J, Chernyyavsky AI Kist DA, Zelickson BD, Lawry MA, Grando SA (2001) Programmed cell death of keratinocytes culminates in apoptotic secretion of a humectant upon secretagogue action of acetylcholine. J Cell Sci 114:1189-1204.

Reeh PW (1986) Sensory receptors in mammalian skin in an in vitro preparation. Neurosci Lett 66:141-146.

Reeh PW (1988) Sensory receptors in mammalian skin-nerve in vitro preparation. In: Progress in brain research. Transduction and cellular mechanisms in sensory receptors (Hamann W, Iggo A, eds), pp 271276. Amsterdam: Elsevier.

Steen KH, Reeh PW (1993) Actions of cholinergic agonists and antagonists on sensory nerve endings in rat skin, in vitro. J Neurophysiol 70:397-405.

Tata AM, Plateroti M, Cibati M, Biagioni S, Augusti-Tocco G (1994) Cholinergic markers are expressed in developing and mature neurons of chick dorsal root ganglia. J Neurosci Res 37:247-255.

Tata AM, Vilaro MT, Mengod G (2000) Muscarinic receptor subtypes expression in rat, chick dorsal root ganglia. Brain Res Mol Brain Res 82:1-10.

Wanke E, Bianchi L, Mantegazza M, Guatteo E, Mancinelli E, Ferroni A (1994) Muscarinic regulation of $\mathrm{Ca}^{2+}$ currents in rat sensory neurons: channel and receptors types, dose-response relationships and cross-talk pathways. Eur J Neurosci 6:381-391.

Weidner C, Schmelz M, Schmidt R, Hansson B, Handwerker HO, Torebjork HE (1999) Functional attributes discriminating mechanoinsensitive and mechano-responsive $\mathrm{C}$ nociceptors in human skin. J Neurosci 19:10184-10190.

Wess J (1996) Molecular biology of muscarinic acetylcholine receptors. Crit Rev Neurobiol 10:69-99.

Wessler I, Reinheimer T, Klapproth H, Schneider FJ, Racke K, Hammer R (1997) Mammalian glial cells in culture synthesize acetylcholine Naunyn Schmiedebergs Arch Pharmacol 356:694-697.

Yang LC, Chen LM, Wang CJ, Buerkle H (1998) Postoperative analgesia by intra-articular neostigmine in patients undergoing knee arthroscopy. Anesthesiology 88:334-339. 